\title{
Random Drug Testing of Physicians: A Complex Issue Framed in 7 Questions
}

\author{
Ethan Cumbler, MD ${ }^{1,2 \star}$; Jean S Kutner, MD, MPSH',2
}

'University of Colorado Hospital, Aurora, Colorado; ${ }^{2}$ University of Colorado School of Medicine, Aurora, Colorado.

hould physicians be subject to random drug testing? It's a controversial topic. One in 10 Americans suffer from a drug use disorder at some point in their lives. ${ }^{1}$ Although physicians engaging in drug diversion is very rare, we recognize, in the context of rising rates of opiate use, that drug misuse and addiction can involve physicians. ${ }^{2,3}$ When it occurs, addiction can drive behaviors that endanger both clinicians and patients. Media reports on drug diversion describe an anesthesiologist who died of overdose from diverted fentanyl and a surgical technician with HIV who used and replaced opioids in the operating room, resulting in thousands of patients needing to be tested for infection. ${ }^{4}$ Multiple outbreaks of hepatitis $C$ involving more than a dozen hospitals in eight states were traced to a single healthcare provider diverting narcotics. ${ }^{5}$ An investigation of outbreaks at various medical centers in the United States over a 10-year period identified nearly 30,000 patients that were potentially exposed and more than 100 iatrogenic infections. ${ }^{6}$

The profession of medicine holds a special place in the esteem of the public, with healthcare providers being among the most trusted professions. Patients rely on us to keep them safe when they are at their most vulnerable. This trust is predicated on the belief that the profession of medicine will self-regulate. Drug diversion by clinicians is a violation of this trust.

Our hospital utilizes existing structures to address substance use disorder; such structures include regular education on recognizing impairment for the medical staff, an impaired clinician policy for suspicion of impairment, and a state physician health program that provides nonpunitive evaluation and treatment for substance use by clinicians. In response to the imperative to mitigate the potential for drug diversion, our health system undertook a number of additional initiatives. These initiatives, included inventory control and tracking of controlled substances and random testing and trigger-based audits of returned medications to ensure the entire amount had been accounted for. As part of this system-wide initiative, UCHealth began random drug testing of employees in safety-sensitive positions (for whom impairment would represent the potential for harm

*Corresponding Author: Ethan Cumbler, MD; E-mail: Ethan.Cumbler@ucdenver.edu; Telephone: 720-848-4289

Published online first October 31, 2018

Received: July 27, 2018; Revised: September 7, 2018;

Accepted: September 16, 2018

() 2019 Society of Hospital Medicine DOI 10.12788/jhm.3099 to others). Medical staff are not employees of the health system and were not initially subject to testing. The key questions at the time included the following:

- Is our organization doing everything possible to prevent drug diversion?

- If nurses and other staff are subject to random drug testing, why would physicians be exempt?

The University of Colorado Hospital (UCH) is the academic medical center within UCHealth. The structure of the relationship between the hospital and its medical staff requires the question of drug testing for physicians to be addressed by the UCH Medical Board (Medical Executive Committee). Medical staff leadership and key opinion leaders were engaged in the process of considering random drug testing of the medical staff. In the process, medical staff leadership raised additional questions about the process of decision making:

- How should this issue be handled in the context of physician autonomy?

- How do we assure the concerns of the medical staff are heard and addressed?

The guiding principles considered by the medical staff leadership in the implementation of random drug testing included the following: (1) as a matter of medical professionalism, for random drug testing to be implemented, the medical staff must elect to submit to mandatory testing; (2) the random drug testing program must be designed to minimize harm; and (3) the process for random drug testing program design needs to engage front-line clinicians. This resulted in a series of communications, meetings, and outreach to groups within the medical staff.

From front-line medical staff members, we heard overwhelming consensus for the moral case to prevent patient harm resulting from drug diversion, our professional duty to address the issue, and the need to maintain public trust in the institution of medicine. At the same time, medical staff members often expressed skepticism regarding the efficacy of random drug testing as a tactic, concerns about operational implementation, and fears regarding the unintended consequences:

- How strong is the evidence that random drug testing prevents drug diversion?

- How can we be confident that false-positive tests will not cause innocent clinicians to be incorrectly accused of drug use?

The efficacy of random drug testing in preventing drug diversion is not settled. The discussion of how to proceed in the absence of well-designed studies on the tactic was robust. One common principle we heard from members of the medical staff was that our response be driven by an authentic organizational desire to reduce patient harm. They expressed 
that the process of testing needs to respect the boundaries between work and home life and to avoid the disruption of clinical responsibilities. Whether targeting testing to "higher risk" groups of clinicians is appropriate and whether or not alcohol and/or marijuana would be tested came up often.

Other concerns expressed also included the intrusion of the institution into the private medical conditions of the medical staff members, breach of confidentiality, or accessibility of the information obtained as a result of the program for unrelated legal proceedings. One of the most prominent fears expressed was the possible impact of false-positive tests on the clinicians' careers.

Following the listening tour by the medical staff and hospital leadership and extensive discussions, the Medical Board voted to approve a policy to implement random drug testing. The deliberative process lasted for approximately eight months. We sought input from other healthcare systems, such as the Veterans Administration and Cleveland Clinic, that conduct random drug tests on employed physicians. A physician from Massachusetts General Hospital who led the 2004 implementation of random drug testing for anesthesiologists was invited to come to Colorado to give grand rounds about the experience in his department and answer questions about the implementation of random drug testing at a Medical Board meeting. The policy went into effect January 2017.

The design of the program sought to explicitly address the issues raised by the front-line clinicians. In the interest of equity, all specialties, including Radiology and Pathology, are subject to testing. Medical staff are selected for testing using a random number generator and retained in the random selection pool at all times, regardless of previous selection for testing. Consistent with the underlying objective of identifying drug diversion, testing is limited to drugs at higher risk for diversion (eg, amphetamine, barbiturate, benzodiazepine, butorphanol, cocaine metabolite, fentanyl, ketamine, meperidine, methadone, nalbuphine, opiates, oxycodone, and tramadol). Although alcohol and marijuana are substances of abuse, they are not substances of healthcare diversion and thus are excluded from random drug testing (although included in testing for impairment). Random drug testing is conducted only for medical staff who are onsite and providing clinical services. The individuals selected for random drug testing are notified by Employee Health, or their clinical supervisor, to present to Employee Health that day to provide a urine sample. The involvement of the clinical supervisor in specific departments and the flexibility in time of presentation was implemented to address the concerns of the medical staff regarding harm from the disruption of acute patient care.

To address the concern regarding false-positive tests, an external medical laboratory that performs testing compliant with Substance Abuse and Mental Health Services and governmental standards is used. Samples are split providing the ability to perform independent testing of two samples. The thresholds are set to minimize false-positive tests. Positive results are sent to an independent medical review officer who confidentially contacts the medical staff member to assess for valid prescriptions to explain the test results. Unexplained positive test re- sults trigger the testing of the second half of the split sample.

To address issues of dignity, privacy, and confidentiality, Employee Health discretely oversees the urine collection. The test results are not part of the individual's medical record. Only the coordinator for random drug testing in Human Resources compliance can access the test results, which are stored in a separate, secure database. The medical review officer shares no information about the medical staff members' medical conditions. A positive drug assay attributable to a valid medical explanation is reported as a negative test.

Positive test results, which would be reported to the President of the Medical Staff, would trigger further investigation, potential Medical Board action consistent with medical staff bylaws, and reporting to licensing bodies as appropriate. We recognize that most addiction is not associated with diversion, and all individuals struggling with substance use need support. The medical staff and hospital leadership committed through this process to connecting medical staff members who are identified by random drug testing to help for substance use disorder, starting with the State Physician Health Program.

The Medical Executive Committees of all hospitals within UCHealth have also approved random drug testing of medical staff. We are not the first healthcare organization to tackle the potential for drug diversion by healthcare workers. To our knowledge, we are the largest health system to have nonemployed medical staff leadership vote for the entire medical staff to be subject to random drug testing. Along the journey, the approach of random drug testing for physicians was vigorously debated. In this regard, we proffer one final question:

- How would you have voted?

Disclosures: The authors have nothing to disclose.

\section{References}

1. Grant BF, Saha TD, Ruan WJ, et al. Epidemiology of DSM-5 drug use disorder: results from the National Epidemiologic Survey on Alcohol and Related Conditions-III. JAMA Psychiatry. 2016;73(1):39-47. doi: 10.1001/jamapsychiatry.2015.2132.

2. Oreskovich MR, Shanafelt T, Dyrbye LN, et al. The prevalence of substance use disorders in American physicians. Am J Addict. 2015;24(1):30-38. doi: 10.1111/ajad.12173.

3. Hughes PH, Brandenburg N, Baldwin DC Jr., et al. Prevalence of substance use among US physicians. JAMA. 1992;267(17):2333-2339. doi:10.1001/ jama.1992.03480170059029.

4. Olinger D, Osher CN. Denver Post- Drug-addicted, dangerous and licensed for the operating room. https://www.denverpost.com/2016/04/23/drug-addicted-dangerous-and-licensed-for-the-operating-room/ Published April 23, 2016. Updated June 2, 2016. Accessed June 7, 2018.

5. Federal Bureau of Investigations. Press Release. Former Employee of Exeter Hospital Pleads Guilty to Charges Related to Multi-State Hepatitis C Outbreak. https://archives.fbi.gov/archives/boston/press-releases/2013/former-employee-of-exeter-hospital-pleads-guilty-to-charges-related-to-multistate-hepatitis-c-outbreak. Accessed June 7, 2018.

6. Schaefer MK, Perz JF. Outbreaks of infections associated with drug diversion by US healthcare personnel. Mayo Clin Proc. 2014;89(7):878-887. doi: 10.1016/j.mayocp.2014.04.007

7. Fitzsimons MG, Baker K, Malhotra R, Gottlieb A, Lowenstein E, Zapol WM. Reducing the incidence of substance use disorders in anesthesiology residents: 13 years of comprehensive urine drug screening. Anesthesiology. 2018;129:821-828. doi: 10.1097/ALN.0000000000002348. 